\title{
Possible signals of constrained minimal supersymmetry at a high luminosity Fermilab Tevatron collider
}

\author{
Stephen Mrenna* \\ California Institute of Technology, Pasadena, California 91125

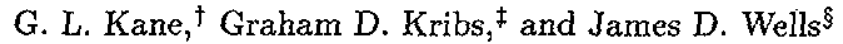 \\ Randall Physics Laboratory, University of Michigan, Ann Arbor, Michigan 48109-1120
}

(Received 15 May 1995)

\begin{abstract}
We study the most promising signals of constrained minimal supersymmetry detectable at a luminosity upgraded $2 \mathrm{TeV}$ Fermilab Tevatron collider. Using a full event-level Monte Carlo program based on PYTHIA and JETSET we simulate the trilepton signal examining in detail the effect of constraints on the parameter space. We also simulate the monolepton and dilepton signals, the $E_{T}+$ jets signal, and the signals of top squark production in supersymmetry all with full standard model backgrounds with realistic detector cuts. We find that large fractions of parameter space can be probed (or eliminated if no signal is found), but mass limits on charginos and neutralinos are not possible based solely on the trilepton signal. Detection efficiencies depend strongly on supersymmetry parameters beyond simply the neutralino and chargino masses; analyses (experimental or theoretical) that do not include this will draw misleading conclusions. Finally, we comment on how searches at CERN LEP II will complement searches at Fermilab.
\end{abstract}

PACS number(s): 12.60.Jv, 14.80.Ly

\section{INTRODUCTION}

The search for supersymmetry (SUSY) should be one of the primary goals at the Fermilab Tevatron and any future collider planned or in construction. While $e^{+} e^{-}$ colliders generally have the advantage of unambiguously finding or excluding superpartners up to $m \sim \sqrt{s} / 2$, their mass reach is strictly energy limited. The CERN $e^{+} e^{-}$collider LEP II, the highest energy $e^{+} e^{-}$collider approved, will be able to probe chargino masses up to about $m_{W}$ but no further. A useful complement to LEP II's important contributions to the search for supersymmetry is provided by a luminosity upgraded Fermilab Tevatron $p \ddot{p}$ collider at $\sqrt{s}=2 \mathrm{TeV}$. As we shall subsequently describe, if the correct supersymmetric theory has a chargino mass below $m_{W}$, the upgraded Tevatron might possibly fail to discover it (hence, the importance of LEP II); however, a high-luminosity Tevatron does have the capability of discovering supersymmetry at mass scales far exceeding the capabilities of LEP IY.

In this paper we report the results of our simulations of many supersymmetric signals for a $p \bar{p}$ collider at $\sqrt{s}=2$ $\mathrm{TeV}$. To this end, we introduce an implementation of all tree-level minimal supersymmetric standard model (MSSM) processes and decay modes in the event generators PYTHIA and JETSET [1-3]. This implementation

*Electronic address: mrenna@cithex.cithep.caltech.edu

†Electronic address: gkane@umich.edu

†Electronic address: kribs@umich.edu

§Electronic address: jwells@walden.physics.lsa.umich.edu of the MSSM is not only a cross check of ISASUSY [4] (see also [5]), which uses the ISAJET [6] generator, but includes the refinements inherent to the PYTHIA-JETSET system. The full details of the event generator will be published in a forthcoming technical report [7].

An equally important task is to run the simulations on supersymmetric solutions, which could be the correct theory of nature. Therefore, we have simulated supersymmetric events in the context of the constrained minimal supersymmetric standard model (CMSSM) [8]. Briefly, the CMSSM enforces gauge coupling unification at the "unification" scale $\sim 10^{16} \mathrm{GeV}$, enforces proper electroweak symmetry breaking, assumes common scalar masses $m_{0}$, common gaugino masses $m_{1 / 2}$, and common trilinear scalar soft couplings $A_{0}$ at the unification scale, requires $R$-parity conservation, and imposes all known experimental constraints such as limits on $b \rightarrow s \gamma$ decays, invisible width constraints on $Z \rightarrow \tilde{\chi}_{i}^{0} \tilde{\chi}_{j}^{0}$ at LEP, etc. Each supersymmetric "solution," with all of its masses and mixings, is determined by five input parameters

$$
m_{0}, m_{1 / 2}, A_{0}, \tan \beta \text {, and } \operatorname{sgn}(\mu) .
$$

We use $m_{t}=170 \mathrm{GeV}$ and have sampled the parameter space as follows: $m_{0}$ is sampled logarithmically from 1 to $1000 \mathrm{GeV}, m_{1 / 2}$ is sampled logarithmically from 30 to $1000 \mathrm{GeV}, A_{0} / m_{0}$ is sampled linearly from -3 to 3 , $\tan \beta$ is sampled by a power law $\left(x^{2}\right)$ for the perturbative ranges from about 1.5 to about 60 , and the sign of $\mu$ is positive or negative with equal probability. Our convention is that $\tan \beta=v_{u} / v_{d}$, where $v_{u}\left(v_{d}\right)$ is the vacuum expectation value that gives mass to the up-type (downtype) fermions. Our convention for the relative sign of $\mu$ is reflected by our choice of the chargino mass matrix 
element $X_{22}=-\mu$ and the neutralino mass matrix elements $Y_{34}=Y_{43}=+\mu$ (which is the opposite convention from Haber and Kane [9]).

The requirements we impose on our supersymmetric solutions are consistent and interrelated. While it is not absolutely necessary for nature to follow all of our theoretical assumptions (such as common scalar masses), we do wish to emphasize that each requirement supports the other requirements to some degree. For example, the experimental requirements such as limits on flavor changing neutral currents support, although do not absolutely require, the theoretical preference in supergravity that all scalars have a common mass at the high scale. Similarly, gauge-coupling unification supports the notion of $R$-parity conservation $[10,11]$, implying an absolutely stable lightest supersymmetric particle (LSP), which is the lightest neutralino $\tilde{\chi}_{1}^{0}$. Proper electroweak symmetry breaking, which is meticulously enforced in the CMSSM, gives the LSP the right properties to be a natural weakly interacting cold dark matter particle, explaining many astrophysical observations [12].

One of the strengths of the CMSSM is that it is strongly constrained and theoretically restrictive. We believe it is remarkable progress that it is possible to construct consistent supersymmetric models that incorporate all the above constraints and provide rich phenomenological predictions. In general, it is always appropriate to study the simplest (hence minimal) theory that is consistent with what is known from the standard model (SM), while simultaneously extending our understanding of nature. The CMSSM is minimal in the sense that it has the spectrum and group structure of the SM plus superpartners, while implementing the minimal supergravity boundary conditions at the unification scale.

Using the CMSSM framework, we have performed event-level analyses of the chargino-neutralino trilepton signal, the chargino-chargino and slepton-slepton dilepton signals, the slepton-sneutrino and chargino-LSP monolepton signals, the squark or gaugino $\not_{T}+$ jets signal, and the signals from stop production and top decays to stop assuming various integrated luminosity scenarios $\left(200 \mathrm{pb}^{-1}, 2 \mathrm{fb}^{-1}\right.$, and $\left.25 \mathrm{fb}^{-1}\right)$ for an upgraded Fermilab Tevatron collider. There have been several previous studies of supersymmetric signals $[13,14]$, all of which are useful and demonstrate the possibility of detecting supersymmetric signals at Fermilab but often make unrealistic assumptions about supersymmetry or make simplifications in the simulation of the signal and background. This study is more general; we find several results different from other studies that stem from two basic principles unique to our approach. First, we only consider values of SUSY parameters that are consistent with the CMSSM. Second, within this general framework, we examine all parameters rather than a set of special cases.

It is possible to construct supersymmetric frameworks that modify some of the theoretical assumptions, and this may be necessary in the future. However, since we sample the supersymmetric parameter space with thousands of solutions, it is likely that many alternatives will lie in regions we have already covered in this study. Hence, the conclusions of this paper are not likely to be signif- icantly modified. In fact, there are already some hints from LEP data that suggest that going beyond the minimality of the CMSSM is necessary because of the $R_{b}$ and $\alpha_{s}$ measurements [15-17]. If these measurements are manifestations of nonminimal supersymmetry, then we expect very light charginos and top squarks to exist, and therefore the Fermilab Tevatron will have a much greater chance to discover them.

We organize this paper as follows. In Sec. II we briefly explain the event simulation needed for every allowed solution in the CMSSM parameter space. Section III contains the motivation and results for each signal we have studied. We perform a full background analysis on every signal, specifying the cuts needed to reduce backgrounds. From the background estimates we determine the detectability or significance of each signal for particular integrated luminosities. In Sec. III A we examine monolepton and dilepton signals, in Sec. IIIB we examine the trilepton signal, and in Sec. IIIC we examine in detail the effect of constraints on supersymmetric parameter space with trilepton detection as an example. In Sec. IIID we examine the $\not_{T}+$ jets signal, in Sec. IIIE we examine the signals from top squark production and in Sec. IIIF we comment on the signal from top decays to the top squark. In Sec. IV we discuss going beyond the CMSSM, including possible effects on supersymmetric signals and detection. Finally, in Sec. V we conclude with a summary of our results and a brief discussion of how a high-luminosity Fermilab Tevatron would complement LEP II.

\section{MONTE CARLO SIMULATION}

Each solution defined by the supersymmetric parameters $m_{0}, m_{1 / 2}, A_{0}, \tan \beta, \operatorname{sgn}(\mu)$ is considered a complete potential theory of nature with well-defined low-energy (weak scale) masses, couplings, etc., obtained from running the masses and couplings from the unification scale to the weak scale. All of these low-energy parameters must be set for each solution. Then, events are generated using 2-to-2 cross section formulas [18-20] incorporated into PYTHIA, which also generates initial and final state QCD and QED radiation. Each 2-to-2 process is derived from a $p \bar{p}$ collision using the CTEQ2L structure functions. The sparticle decays are based on twobody and three-body formulas for the decay rates [19-21] added to JETSET, which performs string fragmentation and hadronization. The final output of the event generator is a list of "stable" particles and their four-momenta. These particles are then fed into a model detector, which smears momenta based on Gaussian energy resolution functions and defines jets based on $E_{T}$ towers with $\phi \times \eta$ segmentation. The detector-level variables are used to define the experimental quantities upon which kinematic cuts are based.

Our model detector is based loosely on the Collider Detector at Fermilab (CDF) [22]. The calorimeter is segmented $\Delta \phi \times \Delta \eta=0.1 \times 0.1$ with $\eta$ coverage to $\eta=4.2$. The hadronic energy resolution is chosen so 
that the jet resolution is roughly $0.7 / \sqrt{E}$ with a degradation to $1.4 \sqrt{E}$ for large $\eta$. The electromagnetic energy resolution is $0.2 / \sqrt{E}$, and the muon momentum resolution is $\sigma_{p_{T}} / p_{T}=\sqrt{\left(0.0009 p_{T}\right)^{2}+(0.0066)^{2}}$. Electrons, muons, and jets are identified for $\eta<2.5$, though the whole calorimeter is used to define the $\vec{H}_{T}$ vector. Isolated leptons $(l)$ are defined as electrons and muons with $E_{T}^{\text {extra }}=\sum_{i} E_{T}^{(i)}-E_{T}^{(l)}<2 \mathrm{GeV}$ within a cone $R \equiv \sqrt{\Delta \phi^{2}+\Delta \eta^{2}} \leq 0.4$ around the lepton. The sum $i$ is over all leptons, photons, and hadrons. Jets $j$ are defined with $R=0.6$ and $E_{T}^{j}>15 \mathrm{GeV}$.

Every signal must go through a process where thousands of supersymmetric solutions are simulated each with thousands of events. The final result is a set of observables (cross sections, decay rates), which we analyze in the form of scatter plots with each point representing a possible supersymmetric theory of nature.

\section{SUPERSYMMETRIC SIGNALS AND BACKGROUNDS}

We consider several potential signals and backgrounds for the Fermilab Tevatron $p \bar{p}$ collider at $\sqrt{s}=2 \mathrm{TeV}$ in the following sections organized by the particular detection signature. In Sec. III C we have performed a detailed analysis of the effect of constraints on the supersymmetric parameter space with the trilepton signal as our example.

\section{A. Monolepton and dilepton signals and backgrounds}

We have simulated the monolepton signals from $\tilde{l}_{L} \tilde{\nu}_{L}, \tilde{\chi}_{1}^{ \pm} \tilde{\chi}_{1}^{0}$ production and the dilepton signals from $\tilde{\chi}_{1}^{ \pm} \tilde{\chi}_{1}^{\mp}, \tilde{\chi}_{2}^{0} \tilde{\chi}_{1}^{0}, \tilde{l}_{L} \tilde{l}_{L}^{*}$, and $\tilde{l}_{R} \tilde{l}_{R}^{*}$ production [23-27]. Of course, any monolepton signal must compete against the huge background from $W^{ \pm}\left(\rightarrow l^{ \pm} \nu\right)$ production, which is $\sim 1 \mathrm{nb}$. For the supersymmetric signals, the energy spectrum of the lepton from the two-body decay of the $\tilde{l}$ or the (two-body or three-body) decay of the $\tilde{\chi}_{1}^{ \pm}$is generally soft because most of the energy is carried by the superpartner decay product $\tilde{\chi}_{1}^{0}$. In addition, the classic $\not_{T}$ signature for SUSY does not easily distinguish signal from background. In the end, we could find no set of straightforward cuts that could reliably extract a monolepton SUSY signal at the Fermilab Tevatron at any luminosity.

The dilepton signals appear much more promising, since the large background from $\gamma^{*}\left(\rightarrow l^{+} l^{-}\right)$and $Z\left(\rightarrow l^{+} l^{-}\right)$can be significantly reduced with an $\not_{T}$ cut and an invariant mass cut on opposite sign leptons near the $Z$ mass. However, the background from the smaller $W^{ \pm}\left(\rightarrow l^{ \pm} \nu\right) W^{\mp}\left(\rightarrow l^{\mp} \nu\right)$ production is virtually irreducible at a total leptonic cross section of $550 \mathrm{fb}$ (without any cuts). We applied a minimal set of cuts and found the dilepton signals from $\tilde{\chi}_{2}^{0} \tilde{\chi}_{1}^{0}, \tilde{l}_{L} \tilde{l}_{L}^{*}$, and $\tilde{l}_{R} \tilde{l}_{R}^{*}$ are ex- tremely difficult to pull out of the $W^{ \pm} W^{\mp}$ background of roughly $\sim 140 \mathrm{fb}$ (with our cuts). Only the $\tilde{\chi}_{1}^{ \pm} \tilde{\chi}_{1}^{\mp}$ signal is visible in a small number of solutions with a chargino mass reach of $m_{\tilde{\chi}_{1}^{ \pm}} \sim 80 \mathrm{GeV}, \sim 110 \mathrm{GeV}$, and $\sim 130 \mathrm{GeV}$ for integrated luminosities of $200 \mathrm{pb}^{-1}, 2$ $\mathrm{fb}^{-1}$, and $25 \mathrm{fb}^{-1}$. No limits on chargino masses could be extracted even at an integrated luminosity of $25 \mathrm{fb}^{-1}$. It is possible that some of the solutions with detectable trilepton signals described in the next section could be confirmed by the dilepton signal [27].

\section{B. Trilepton signal and backgrounds}

The trilepton signal from $\tilde{\chi}_{1}^{ \pm} \tilde{\chi}_{2}^{0}$ production is probably the most promising signal of supersymmetry at a hadron collider [28,30,25-27]. Although the signal is obtained from the chargino decaying to one lepton and the neutralino decaying to two leptons, as in the preceding section, the resulting trilepton signature has two clear advantages over the monolepton and dilepton signals. First, the correlations induced between the mass and mixing parameters in the CMSSM almost invariably output a lightest chargino, which is mostly a charged $W$-ino $\tilde{W}^{t}$ and a second-lightest neutralino, which is largely a neutral $W$-ino $\tilde{W}^{3}$. Thus, the coupling at the $W^{\mp} \tilde{\chi}_{1}^{ \pm} \tilde{\chi}^{0}$ vertex is near maximal in the CMSSM, since it is the supersymmetrized version of the $W^{ \pm} W^{\mp} W^{3}$ standard model vertex. Second and more important, the trilepton signal has few sizable backgrounds, in stark contrast to the monolepton and dilepton signals. In this section we explore all aspects of the trilepton signal, including a full background analysis and a complete examination of the CMSSM parameter space.

There are six sources of physics ${ }^{1}$ backgrounds relevant to the trilepton signal: $W Z, Z Z, t \bar{t}, t \bar{b}+b \bar{t}, Z+g$, and $W+g$. The $W Z, Z Z$, and $t \bar{t}$ backgrounds have been studied before (see, e.g., Refs. $\{29,30,25]$ ). Our results are similar but more general, since we study both the traditional backgrounds (first three) and also backgrounds from the leptonic decays of $b$ quarks including those from $g^{*} \rightarrow b \vec{b}$ splitting. The latter can be important, especially if one considers measuring leptons with low $p_{T} \approx 5 \mathrm{GeV}$, as we do in this study. In general, we have computed the backgrounds to an accuracy such that statistical fluctuations will not affect our conclusions. For the $W Z, Z Z$, and $Z+g$ backgrounds, we properly incorporate the effect of $\gamma^{*}$ interference in $Z$ production and use the full $2 \rightarrow 4$ matrix elements inherent in the pYrHIA Monte Carlo generator. We also find it is essential to simulate all possible decay channels of gauge bosons that could possibly lead to leptons in the final state (i.e., we include

\footnotetext{
${ }^{1}$ Detectors have additional backgrounds from, for example, $\gamma$ 's that "fake" an electron. The dominant background where this can occur is for $\gamma^{*} / Z \rightarrow \tau^{+} \tau^{-} \rightarrow e^{\prime}$ s/ $\mu$ 's with a $\gamma$ (and $\vec{B}_{x}$ ) in the final state. We find that at a "fake" rejection rate $\lesssim 10^{-4}$ our set of cuts effectively eliminates this background.
} 
the $\tau$ decay channels). In the end, we find the $W Z$ and $Z Z$ backgrounds are the most difficult to eliminate, since they contain real isolated leptons (distinguishable from leptons from heavy quark decay, which are generally not isolated from hadrons).

Below, we enumerate the kinematic cuts applied and the physics motivation behind them.

(1) Three isolated leptons (electrons or muons), no hard jets, to reduce QCD backgrounds with $p_{T}$ cuts of $\left(p_{T}^{(1)} ; p_{T}^{(2)} ; p_{T}^{(3)}\right)>(10 ; 5 ; 5) \mathrm{GeV}$ for the highest $(1)$, nexthighest (2), and lowest (3) $p_{T}$ leptons. $p_{T}^{(1)}>10 \mathrm{GeV}$ is necessary for triggering.

(2) $\left|m_{l^{+} l^{-}}-m_{Z}\right|>15 \mathrm{GeV}$ for the opposite sign, same flavor, lepton pair invariant masses to reduce $Z \rightarrow$ $e^{ \pm} e^{\mp}, \mu^{ \pm} \mu^{\mp}$ backgrounds.

(3) $m_{l l^{\prime}}>20 \mathrm{GeV}$ for all lepton pair invariant masses to reduce $\gamma^{*}, b \rightarrow l+X$.

(4) The opposite sign $l^{(1)}, l^{(2)}$ (highest and nexthighest $p_{T}$ leptons) must not be "back to back": $\mid \phi_{2}-$ $\phi_{1} \mid<2.5$ to reduce $Z \rightarrow \tau^{ \pm} \tau^{\mp}$ background.

(5) Transverse mass is required to be $m_{T}<70 \mathrm{GeV}$ to reduce $W$ backgrounds, where $m_{T}$ is constructed from $l^{(1)}$ 's momentum and the $\vec{H}_{T}$ vector.

Table I shows the reduction of backgrounds as a function of the cuts $1-5$. In addition, we find no backgrounds from $W+g$ or $t \bar{b}+b \bar{t}$, which would require two heavy quarks to decay to isolated leptons. This reinforces our confidence that we need only consider the background from a single heavy-quark decay to an isolated lepton. After applying all of the cuts listed above, our final trilepton background estimate is $0.67 \mathrm{fb}$ for the Fermilab Tevatron $p \bar{p}$ collider at $\sqrt{s}=2 \mathrm{TeV}$.

Using this background estimate, we can calculate the smallest SUSY trilepton cross section, folded with the leptonic branching ratios and detection efficiency (denoted $\sigma \times \mathrm{BR} \times \mathrm{EFF}$ ) that is detectable above backgrounds. We find the minimum $\sigma \times \mathrm{BR} \times \mathrm{EFF}$ for integrated luminosities of $200 \mathrm{pb}^{-1}, 2 \mathrm{fb}^{-1}$, and $25 \mathrm{fb}^{-1}$ at the Fermilab Tevatron to be $25 \mathrm{fb}, 3.0 \mathrm{fb}$, and 0.82 $\mathrm{fb}$, respectively, based on the larger of five events or the number of events required for a $5 \sigma$ signal over the square root of the background.

In Fig. 1 we have plotted the total supersymmetric $\sigma \times \mathrm{BR} \times \mathrm{EFF}$ from $\tilde{\chi}_{1}^{ \pm} \tilde{\chi}_{2}^{0}$ production (including all diagrams) versus the lightest chargino mass for well over 2000 solutions spanning the CMSSM parameter space up to $m_{\tilde{\chi}_{1}^{ \pm}} \leq 500 \mathrm{GeV}$. Note that a tail of CMSSM solutions

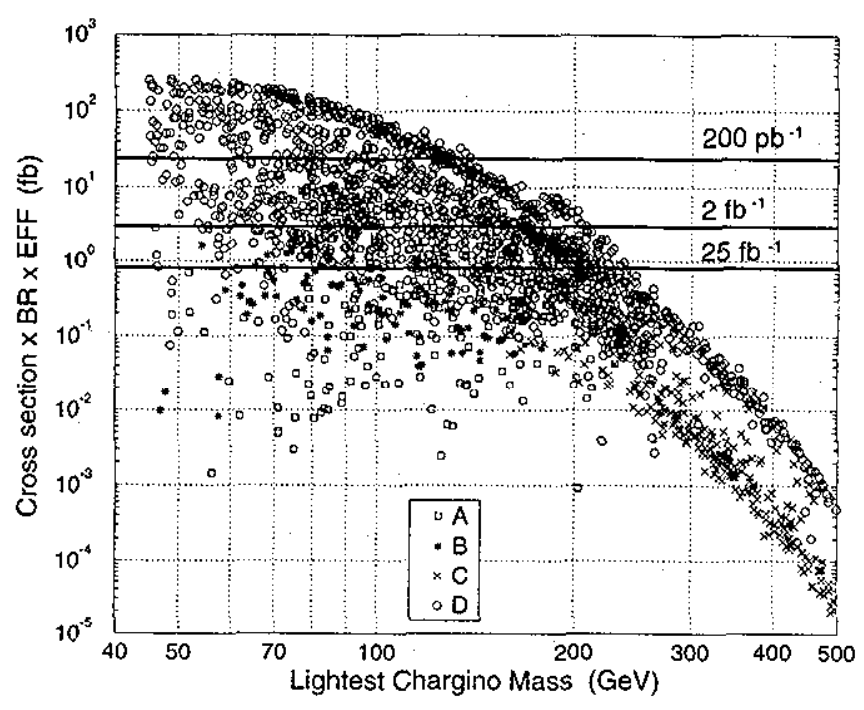

FIG. 1. Total supersymmetric trilepton signal $(\sigma \times \mathrm{BR} \times \mathrm{EFF})$ after cuts versus the lightest chargino mass in the CMSSM. The branching ratio (BR) is defined as the fraction of $\tilde{\chi}_{1}^{ \pm} \tilde{\chi}_{2}^{0}$ events that decay to three leptons. The efficiency (EFF) is defined as the fraction of three-lepton events that pass the cuts described in the text. The minimum detectable $\sigma \times \mathrm{BR} \times \mathrm{EFF}$ for integrated luminosities of 200 $\mathrm{pb}^{-1}, 2 \mathrm{fb}^{-1}$, and $25 \mathrm{fb}^{-1}$ is shown by the dark horizontal lines at $25 \mathrm{fb}, 3.0 \mathrm{fb}$, and $0.82 \mathrm{fb}$, respectively. The different symbols refer to solutions showing interesting behavior where the second lightest neutralino $\tilde{\chi}_{2}^{0}$ has $(A)$ a neutral "invisible" branching ratio (generally $\tilde{\chi}_{2}^{0} \rightarrow \tilde{\nu}_{L} \bar{\nu}$ then $\tilde{\nu}_{L} \rightarrow \tilde{\chi}_{1}^{0} \nu$ ) $>90 \%$, $(B)$ a large destructive interference in three-body leptonic decays defined by $R_{\text {interference }}<0.1$ (see Fig. 2), $(C)$ a branching ratio to Higgs bosons $>50 \%$ dominates, or $(D)$ all other solutions.

does exist for $m_{\tilde{\chi}_{1}^{ \pm}}>500 \mathrm{GeV}$, but we do not consider them here. Each symbol represents one solution defined by $m_{0}, m_{1 / 2}, A_{0}, \tan \beta$, and $\operatorname{sgn}(\mu)$. Each solution, when the masses and couplings are run from the unification scale to the weak scale, has its own well-defined masses, mixings, branching ratios, etc. The chargino mass represents one of these well-defined weak scale observables that is directly related to the production cross section (hence, our choice of $x$ axis). In addition, the branching ratios of the superpartners also represent weak scale observables that are crucial to a correct calculation of the

TABLE I. Summary of our background studies of the supersymmetric trilepton signal. Cuts 1-5 are described in the text. The final background estimate using our cuts is $0.67 \mathrm{fb}$.

\begin{tabular}{lccccc}
\hline \hline & \multicolumn{5}{c}{ Background $\sigma$ after cuts (fb) } \\
\multicolumn{1}{c}{ Process } & Cut 1 & Cuts 1 and 2 & Cuts 1-3 & Cuts 1-4 & Cuts 1-5 \\
\hline$W Z, W \gamma^{*}$ & 22.6 & 1.3 & 1.0 & 0.85 & 0.38 \\
$Z Z, Z \gamma^{*}, \gamma^{*} \gamma^{*}$ & 5.3 & 0.22 & 0.16 & 0.12 & 0.09 \\
$t \bar{t}$ & 0.42 & 0.33 & 0.23 & 0.17 & 0.06 \\
$Z+g, \gamma^{*}+g$ & 5.0 & 0.64 & 0.23 & 0.14 & 0.14 \\
Total & & & & & 1.28 \\
\hline
\end{tabular}


trilepton signal. All decay channels of all possible superpartners that could be produced directly $\left(\tilde{\chi}_{1}^{ \pm}, \tilde{\chi}_{2}^{0}\right)$, or as a result of decays (gauginos, sleptons, and Higgs), must be computed for each solution.

The second-lightest neutralino's leptonic branching ratio can sometimes be small enough to prevent a detectable trilepton signal even at low neutralino (or chargino) masses. Here we elaborate on the neutralino $\left(\tilde{\chi}_{2}^{0}\right)$ branching ratios, while examining in detail the solutions identified in Fig. 1 that have a small leptonic branching ratio. (A) The $\tilde{\chi}_{2}^{0}$ often decays predominantly to neutral ("invisible") products through the two-body $\tilde{\nu}_{L} \bar{\nu}$ channel followed by $\tilde{\nu}_{L} \rightarrow \tilde{\chi}_{1}^{0} \nu$, or directly through the three-body $\tilde{\chi}_{1}^{0} \nu \bar{\nu}$. We have identified these solutions in Fig. 1 with separate symbols to show clearly that they are a source of some low $\sigma \times \mathrm{BR} \times \mathrm{EFF}$ solutions. (B) The three-body leptonic decays of the $\tilde{\chi}_{2}^{0}$ can have destructive interference among the diagrams mediated by the $\tilde{l}_{L}, \tilde{l}_{R}$, and $Z$. This interference effect has also been observed in Ref. [27]. Once again, we identify such solutions in Fig. 1 with a separate symbol. Numerically, we found that significant destructive interference occurs only for chargino masses $m_{\tilde{\chi}_{1}^{ \pm}} \lesssim 200 \mathrm{GeV}$. Generally, the interference is characterized by $m_{\tilde{l}_{L}}, m_{\tilde{l}_{R}} \sim 200 \mathrm{GeV}$. At lower slepton masses, it is the slepton-mediated three-body decays that dominate the decay width of $\tilde{\chi}_{2}^{0}$ into leptons. At higher slepton masses, the $Z$-mediated three-body decays dominate. This is demonstrated in Fig. 2, where the ratio of the squared-amplitude with and without the interference

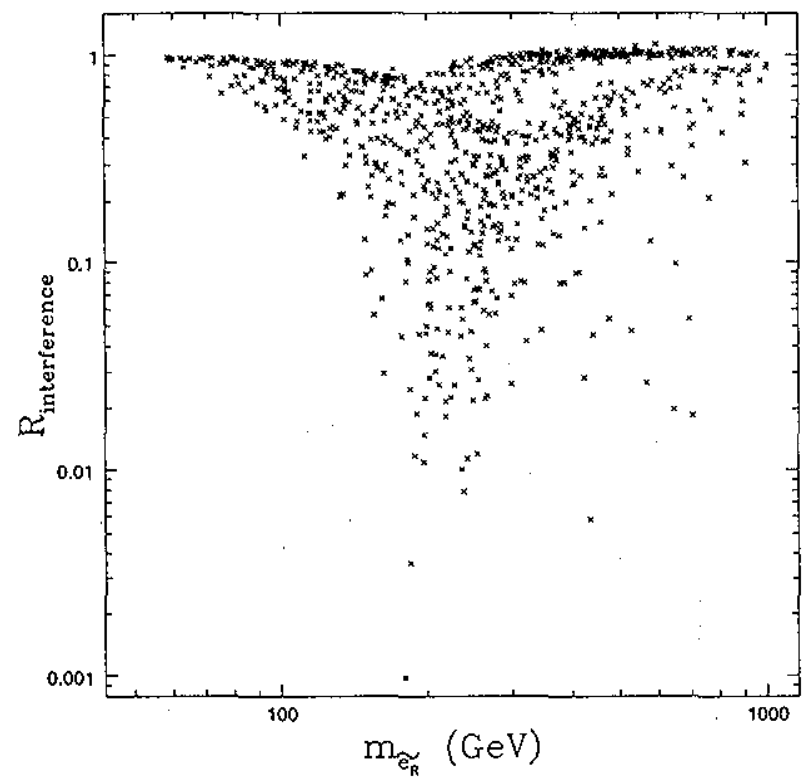

FIG. 2. To illustrate the effects of interference in the three-body decays $\tilde{\chi}_{2}^{0} \rightarrow \tilde{\chi}_{1}^{0} l^{+} l^{-}$, we have plotted the ratio ( $\left.R_{\text {interference }}\right)$ of the full $Z, \tilde{e}_{R}$, and $\tilde{e}_{L}$ squared amplitude to the sum of the squares of the three individual diagrams for the branching ratio to electrons (which is also representative of the branching ratio to muons and taus) against the mass of the right selection. Note that $R_{\text {interference }}$ is expected to be $\sim 1$ if the magnitude of the interference is small and cannot exceed $7 / 3$ with our definition (since there is no interference between $\tilde{e}_{L}$ and $\tilde{e}_{R}$ diagrams). terms for $\tilde{\chi}_{2}^{0} \rightarrow \tilde{\chi}_{2}^{0} e^{+} e^{-}$(which is representative of all the leptonic channels) is plotted versus the right selectron mass for all solutions described above. We find destructive interference for both signs of $\mu$. The interference for $\mu>0$ is largest for $\tan \beta \gtrsim 10$, while the interference for $\mu<0$ occurs over the entire range of $\tan \beta$. Finally, (C) the decays of $\tilde{\chi}_{2}^{0} \rightarrow \tilde{\chi}_{1}^{0} h^{0}$ have been explicitly identified ${ }^{2}$ in Fig. 1. We note that to compute the decay widths of $h^{0}$, one must evaluate all masses (and couplings) at the scale of $m_{h}$ o [31]. Since there are completely general upper mass bounds [32] on $m_{h^{0}} \ll 2 m_{t}$, the only decay channels open to $h^{0}$ are to $b \bar{b}, \tau^{+} \tau^{-}$and lighter particles. When $m_{b}$ and $m_{\tau}$ are evaluated at $m_{h^{0}}$, the leptonic decay width of $h^{0} \rightarrow \tau^{+} \tau^{-}$is about $10 \%$. This has a dramatic effect on the leptonic branching ratio of the $\tilde{\chi}_{2}^{0}$ when both $\tau$ 's decay leptonically to $e$ 's and $\mu$ 's. Further, it is likely that one-prong pion decays from the $\tau$ 's from the Higgs are measurable and would provide an additional single lepton plus two one-prong signal of chargino-neutralino production. However, accurate estimates of one-prong backgrounds are more difficult than leptonic backgrounds, so to be conservative we do not include isolated pions in our signal. The final result is that we find the trilepton signal present and detectable given a high enough integrated luminosity even for large neutralino masses $\left(m_{\tilde{\chi}_{2}^{0}} \sim m_{\tilde{\chi}_{1}^{ \pm}} \gtrsim 250 \mathrm{GeV}\right)$, where the $\tilde{\chi}_{2}^{0} \rightarrow \tilde{\chi}_{1}^{0} h^{0}$ decay channel is open.

Once the cross section and branching ratios are computed, a full event-level simulation was performed using the cuts described above. The cuts undoubtedly have an impact on the detectable signal, and we quantify this by defining the "detection efficiency" EFF, as the ratio of the number of three-lepton events that passed our cuts to the total number of three-lepton events from $\tilde{\chi}_{1}^{ \pm} \tilde{\chi}_{2}^{0}$ production. We find that the efficiency can vary dramatically over the parameter space, as is illustrated in Fig. 3, where we plot the efficiency versus the chargino mass. The structure of the efficiency plot is clear: At low chargino masses $\left(m_{\tilde{\chi}_{1}^{ \pm}} \lesssim 150 \mathrm{GeV}\right)$ most of the $\tilde{\chi}_{1}^{ \pm}, \tilde{\chi}_{2}^{0}$ decays are three-body with a gradual rise in efficiency because of the increasing energy of the leptons. The low-efficiency solutions (EFF $\lesssim 10^{-2}$, where we found the EFF to be $>10^{-4}$ for all solutions) occur when $m_{\tilde{\chi}_{1}^{ \pm}} \approx m_{\tilde{\nu}_{L}}$, the two-body sneutrino modes are open, and there is little energy for the lepton. The neutralino also has analogous problems when $m_{\tilde{\chi}_{2}^{0}} \approx m_{\hat{l}_{L} \text { or } \tilde{l}_{R}}$ so that two-body modes are open with one rather soft lepton. At higher chargino masses $\left(m_{\tilde{\chi}_{1}^{ \pm}} \gtrsim 200 \mathrm{GeV}\right)$, the two-body decays $\tilde{\chi}_{1}^{ \pm} \rightarrow \tilde{\chi}_{1}^{0} W$ and $\tilde{\chi}_{2}^{0} \rightarrow \tilde{\chi}_{1}^{0} Z$ also cause a lower efficiency for the simple reason that our cuts are designed to eliminate real $W$ 's and $Z$ 's. Finally, the somewhat lower efficiency of the $\tilde{\chi}_{2}^{0} \rightarrow \tilde{\chi}_{1}^{0} h^{0}$ mode is visible at high chargino masses $\left(m_{\tilde{\chi}_{1}^{ \pm}} \gtrsim 250 \mathrm{GeV}\right)$, since those

\footnotetext{
${ }^{2}$ Other authors, (see, e.g., Refs. $[30,46]$ ) have identified the importance of the decay mode of $\tilde{\chi}_{2}^{0}$ to $h$, but with somewhat different conclusions.
} 


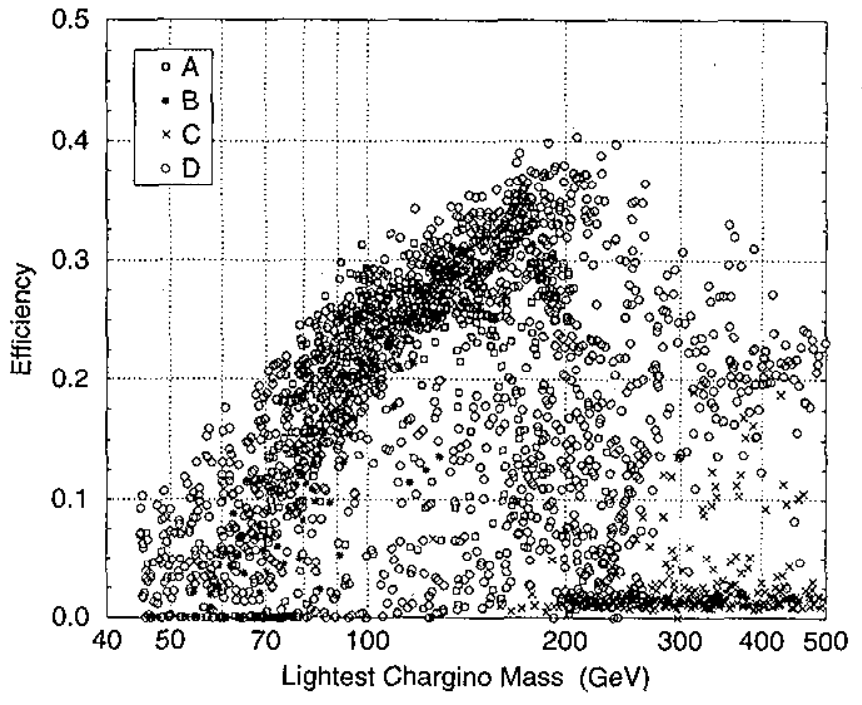

FIG. 3. Detection efficiency of the trilepton signal after cuts versus the lightest chargino mass in the CMSSM. The efficiency is defined as the fraction of three-lepton events that pass the cuts described in the text. The different symbols refer to solutions interesting behavior where the second lightest neutralino $\tilde{\chi}_{2}^{0}$ has $(A)$ a neutral "invisible" branching ratio (generally $\tilde{\chi}_{2}^{0} \rightarrow \tilde{\nu}_{L} \bar{\nu}$ then $\tilde{\nu}_{L} \rightarrow \tilde{\chi}_{1}^{0} \nu$ ) $>90 \%,(B)$ a large destructive interference in three-body leptonic decays defined by $R_{\text {interference }}<0.1$ (see Fig. 2), $(C)$ a branching ratio to Higgs $>50 \%$ dominates, or $(D)$ all other solutions.

events will tend to fail our kinematic cuts more often. Note that the efficiencies described here (visible in Fig. 3) are automatically included in the $\sigma \times \mathrm{BR} \times \mathrm{EFF}$ plot of Fig. 1.

The results from this trilepton analysis are manifest in Fig. 1. The Fermilab Tevatron can probe chargino masses up to $140 \mathrm{GeV}, 210 \mathrm{GeV}$, and $240 \mathrm{GeV}$ with integrated luminosities of $200 \mathrm{pb}^{-1}, 2 \mathrm{fb}^{-1}$, and $25 \mathrm{fb}^{-1}$, respectively. However, the Tevatron cannot set mass limits on charginos or neutralinos from just the trilepton signal. This result follows directly from using the full CMSSM parameter space with the complete two- and three-body branching ratios of superpartners and a full event-level simulation with realistic detector cuts. The requirement for a full simulation of all constrained so- lutions so that the efficiency is calculated correctly for every solution is demonstrated in Fig. 3. Perhaps new experimental or theoretical constraints could eventually eliminate the low-lying solutions in Fig. 1, thereby allowing a lower limit on $m_{\tilde{\chi}_{t}^{t}}$ to be set if no signal is found. Nevertheless, we emphasize that a significant part of the parameter space can be eliminated if no signal is found.

\section{Effects of constraints on supersymmetric parameter space}

Requiring a constrained parameter space that satisfies our theoretical expectations with all current experimental results is very important. To illustrate the effect of constraints on the parameter space, we looked at a particular choice of parameters that would give a qualitative feel for how $b \rightarrow s \gamma$ and relic density cuts in the CMSSM typically impact the trilepton signal. For this example, $\tan \beta=5, A_{0}=0$, and $\operatorname{sgn}(\mu)=+$ (only for illustrative purposes in this section), while the values of $m_{0}$ and $m_{1 / 2}$ were selected randomly on a logarithmic scale up to $1 \mathrm{TeV}$. We applied all CMSSM cuts to the data sample except the $b \rightarrow s \gamma$ and relic density cuts.

In Figs. 4(a) and 4(b) we plot the trilepton signal before the $b \rightarrow s \gamma$ and relic density cuts. The plotting variables are $m_{0}$ (common scalar mass) and $m_{1 / 2}$ (common gaugino mass) in Fig. 4(a) and the more concrete $m_{\tilde{e}_{R}}$ and $m_{\tilde{\chi}_{1}^{ \pm}}$in Fig. 4(b). [Figure $4(\mathrm{~b})$ is nothing more than a direct remapping of all the points in Fig. 4(a).] The $\times$ 's in Figs. 4(a) and 4(b) are solutions that are detectable through the supersymmetric trilepton signal with an integrated luminosity of $25 \mathrm{fb}^{-1}$, while the dots are not detectable without higher integrated luminosity.

In Figs. 5(a) and 5(b) we produce the same type of scatter plots, this time using the $b \rightarrow s \gamma$ observable $[33,8,34,35]$. All solutions marked with a $\times$ are now just those with the additional constraint $B(b \rightarrow s \gamma)<$ $5.4 \times 10^{-4}$. We choose $5.4 \times 10^{-4}$ as our discriminate value, since that is the $95 \%$ upper limit value published by CLEO [36]. CLEO has also recently reported [37] a measurement of

$$
B(b \rightarrow s \gamma)=(2.32 \pm 0.67) \times 10^{-4}
$$
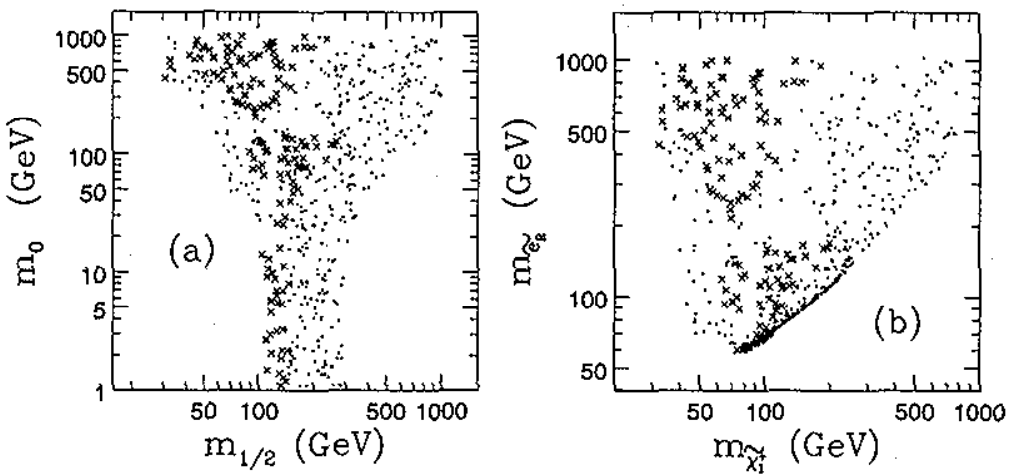

FIG. 4. The trilepton signal before $b \rightarrow s \gamma$ and relic density cuts. The solutions represented by the $X$ 's are detectable with an integrated luminosity of $25 \mathrm{fb}^{-1}$ at the Fermilab Tevatron, while the dots are not. 

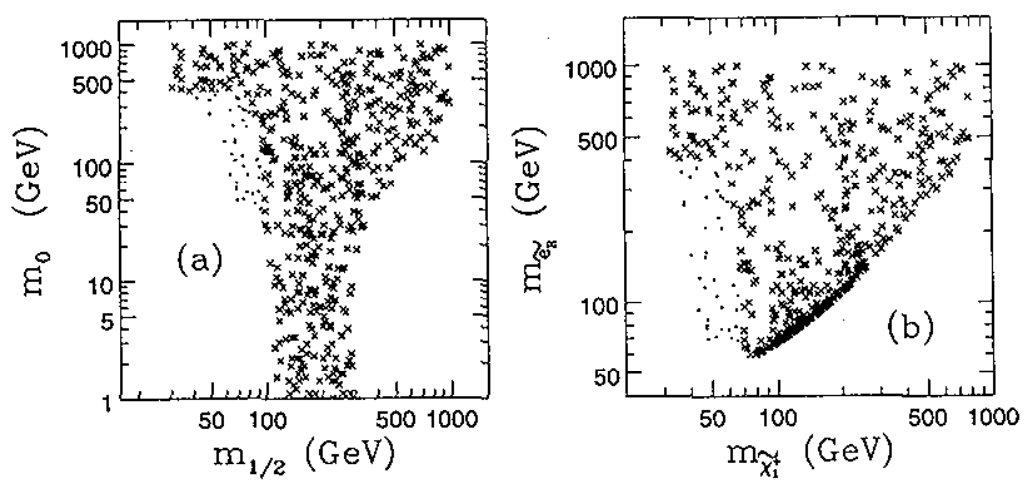

FIG. 5. The effect of $b \rightarrow s \gamma$ cut on the parameter space. The solutions represented by the $X$ 's satisfy the cut $B(b \rightarrow s \gamma)<5.4 \times 10^{-4}$, while the dots do not.
This would suggest that we could add $0.67 \times 10^{-4}$ to the central value and declare this our $1 \sigma$ upper bound on $B(b \rightarrow s \gamma)$, and then insist that all CMSSM solutions have a predicted value of $B(b \rightarrow s \gamma)<2.99 \times 10^{-4}$. However, the QCD uncertainties $\{38\}$ in the $B(b \rightarrow s \gamma)$ calculation (approximately $25 \%$ ) require us to use a higher "calculation upper limit" than the experimental upper limit; therefore, to be conservative we accept all solutions with a calculated $B(b \rightarrow s \gamma)<5.4 \times 10^{-4}$. A significant number of solutions in Figs. 5(a) and $5(\mathrm{~b})$ are cut out by this constraint. Interestingly, many of the solutions cut out by this constraint overlap with light chargino solutions, which were not detectable by the trilepton signal.

The relic density constraint is also an important cut on the CMSSM parameter space. We expect that nature is described by an $R$-parity conserving supersymmetric theory $[10,11]$, and thus the lightest supersymmetric particle (LSP) is absolutely stable. In the early Universe these stable particles were in thermal equilibrium with the photons until the expansion rate of the Universe became roughly equal to their annihilation rate. When this happens, the LSP's fall out of equilibrium with the photons and their relic abundance stabilizes. If this decoupling occurs too soon (weak annihilation rates) then the Universe becomes matter dominated too early. Most observational data indicates that the Universe is more than about 10 billion years old, and this translates into an upper bound on the relic density of LSP's. Quantitatively this upper bound, often called the "age of the Universe constraint," is most often expressed as a condition on $\Omega_{\mathrm{LSP}} h^{2}$ :

$$
\Omega_{\mathrm{LSP}} h^{2}<1.0 \text { (age of the Universe constraint) }
$$

where $h$ is the Hubble parameter. As part of the CMSSM we require $\Omega_{\mathrm{LSP}} h^{2}<1.0$. Except for a small region in parameter space $[39,40]$, the age of the Universe constraint will exclude all SUSY models with large squark and slepton masses $[39,8]$. This can be understood by realizing that the LSP is mostly (but not completely) $B$-ino in the CMSSM, and the $B$-ino has no coupling to the $Z$ boson and therefore no annihilation channels through an $s$-channel $Z$ are accessible. Then the LSP must annihilate through a $t$-channel squark or slepton:

$$
\left\langle\sigma v_{\mathrm{rel}}\right\rangle \propto \frac{1}{m_{\tilde{f}}^{4}}
$$

and since

$$
\Omega_{\mathrm{LSP}} h^{2} \propto \frac{1}{\left\langle\sigma v_{\mathrm{rel}}\right\rangle}
$$

then

$$
\Omega_{\mathrm{LSP}} h^{2} \propto m_{\tilde{f}}^{4}
$$

Therefore, the relic abundance $\Omega_{\text {LSP }}$ grows with the supersymmetric breaking scale. This cutoff on supersymmetric masses depends on all the input parameters but is generally around $1 \mathrm{TeV}$.

The importance of this robust cosmological requirement on studies of supersymmetry detectability cannot be overstated. Any successful constraint on the mass of supersymmetric particles is obviously of great relevance to a collider program trying to discover or rule out supersymmetry. It is for this reason that we have ensured that this constraint is incorporated into all solutions analyzed in this paper.

A lower bound on $\Omega_{\mathrm{LSP}} h^{2}$ can also be obtained from the requirement that the LSP's constitute a significant amount of the Universe's mass fraction to be a viable cold dark matter candidate. Using this requirement, we obtain a quantitative lower bound;

$$
\Omega_{\mathrm{LSP}} h^{2} \gtrsim 0.05 \text { (cold dark matter constraint). }
$$

This cold dark matter constraint is not applied to the CMSSM solutions anywhere else in the paper except in Figs. 6 and 7 , since no experiment has yet confirmed the identity of the cold dark matter. However, we do consider it a major success of the unified supersymmetric theories that a stable weakly interacting massive object with a large relic abundance is generically predicted in accordance with astrophysical observations.

In Figs. 6(a) and 6(b) the $\times$ 's represent solutions that are consistent with both the age of the Universe 

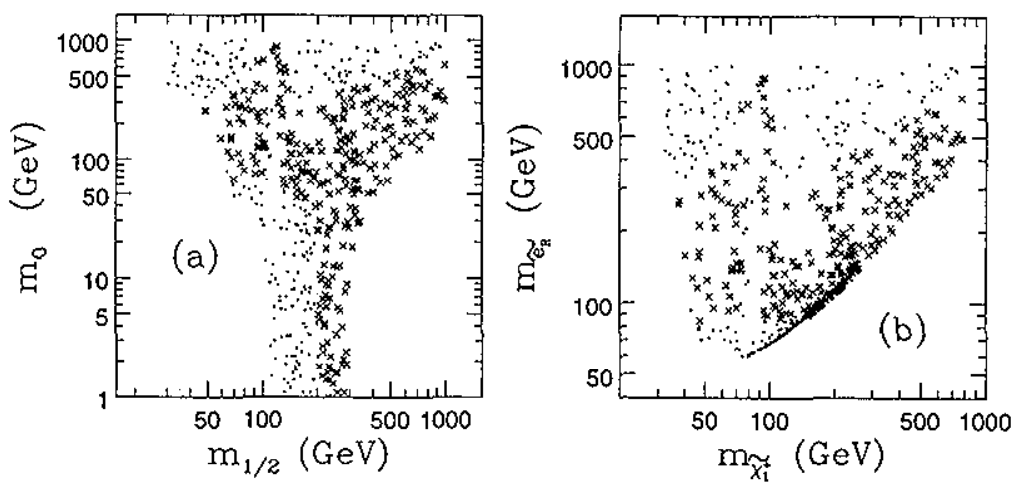

FIG. 6. The effect of the $\Omega_{\mathrm{LSP}} h^{2}>0.05$ cold dark matter cut and $\Omega_{\mathrm{LSP}} h^{2}<1.0$ age of the Universe constraint cuts on the parameter space. The solutions represented by $\times$ 's have $0.05<\Omega_{\mathrm{LSp}} h^{2}<1.0$, while the dots do not. and the cold dark matter constraints, summarized by $0.05<\Omega_{\mathrm{LSP}} h^{2}<1.0$. The $\times$ 's represent all solutions that pass the relic density cut, and the dots represent all solutions that lie outside the cut. The dots in the lower left corners of both Figs. 6(a) and 6(b) are solutions with $\Omega_{\mathrm{LSP}} h^{2}<0.05$, and thus are not interesting dark matter candidates. The dots with high $m_{0}$ and high $m_{\tilde{e}_{R}}$ are solutions that have $\Omega_{\mathrm{LSP}} h^{2}$ in qualitative agreement with the argument given above that large SUSY scalar masses yield large $\Omega_{\mathrm{LSp}} h^{2}$. Some solutions at high $m_{0}$ survive at $m_{1 / 2} \sim 100 \mathrm{GeV}$, where the LSP's annihilate through the $Z$ resonance. Although the coupling to the $Z$ is quite small, the resonance effect is dominant here, and the relic density can stay small.

Finally, Figs. 7(a) and 7(b) are identical to Figs. 4(a) and $4(b)$ except now the $B(b \rightarrow s \gamma)$ and $\Omega_{\mathrm{LSP}} h^{2}$ cuts are included. Many more models are ruled out from the additional cuts, and the resulting parameter space provides a more constrained set of solutions that the Fermilab Tevatron could either detect or rule out (represented by the $\times$ 's). The qualitative shift in the parameter space by including all constraints on the CMSSM demonstrated in this example points to the importance of including all relevant constraints simultaneously on supersymmetric solutions for a realistic study of collider capabilities.

\section{Missing $E_{T}+$ jets as a signal for squarks and gluinos}

A classic signature of supersymmetry is multijet events with a large $\mathbb{H}_{T}[41,42,45]$. This signal can result from many supersymmetric parton-level processes, and we have simulated only a practical subset of these: squarkgluon pair $(\tilde{q} \tilde{q}, \tilde{g} \tilde{g})$, squark+gluino $(\tilde{q} \tilde{g})$, and squark (gluino) +chargino (neutralino) production $(\tilde{q} \tilde{\chi}, \tilde{g} \tilde{\chi})$. In the special case of $m_{1 / 2} \gg m_{0}$, then $m_{\tilde{g}} \approx m_{\tilde{q}}$; otherwise $m_{\tilde{g}} \lesssim m_{\tilde{q}}$, and we expect the dominant signal to be $\tilde{g} \tilde{g}$ production with three-body decays into other gauginos and jets in that region (see Fig. 8). A large potential background is QCD multijet production folded with the intrinsic $\not_{T}$ resolution of the detector. Since jet production is the largest of all high- $p_{T}$ processes at a hadron collider, we need to justify neglecting it, if indeed we can. To estimate the background contribution, we generate all QCD parton-level processes for $\hat{p}_{T}>30$ $\mathrm{GeV}$ with initial and final state $\mathrm{QCD}$ radiation. The $\not_{T}$ resolution $\sigma_{\not l}$ is approximated by the CDF-like formula $\sigma_{\not_{T}^{\prime}}=0.7 \sqrt{\Sigma E_{T}}$, where $\Sigma E_{T}$ is the scalar sum of all the jet transverse energy. The $\mathbb{H}_{T}$ resolution degrades with increased jet activity, so only high- $p_{T}$ events can generate a large $E_{T}$. The contribution of each event is folded with the probability that the measured $\not_{T}$ fluctuates to $\not_{T}^{\text {cut }}$ or more using the Gaussian formula

$$
P\left(\not_{T}>\#_{T}^{\text {cut }}\right)=\frac{2}{\sqrt{\pi}} \int_{t^{\prime}}^{\infty} e^{-t^{2}} d t
$$

where

$$
t^{\prime}=\frac{\not Z_{T}^{\mathrm{cut}}}{\sigma_{\not_{T}^{\prime}} / \sqrt{2}} .
$$
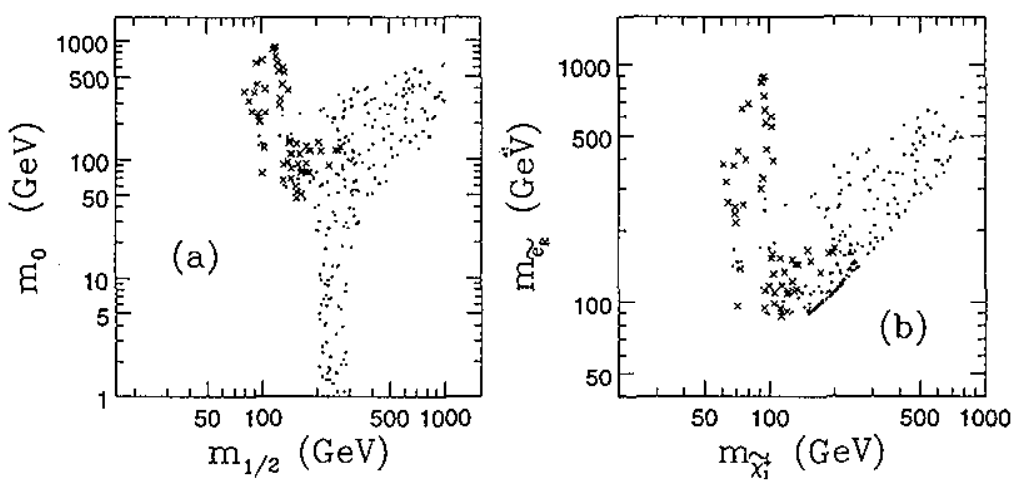

FIG. 7. The trilepton signal is plotted as in Fig. 4 except all solutions ( $x$ 's and dots) must satisfy the $b \rightarrow s \gamma$ cut and relic density cuts described in the text. Note the large regions of parameter space excluded by these cuts, and also the remaining distribution of detectable solutions (represented by $X$ 's for an integrated luminosity of $25 \mathrm{fb}^{-1}$, as in Fig. 4). 


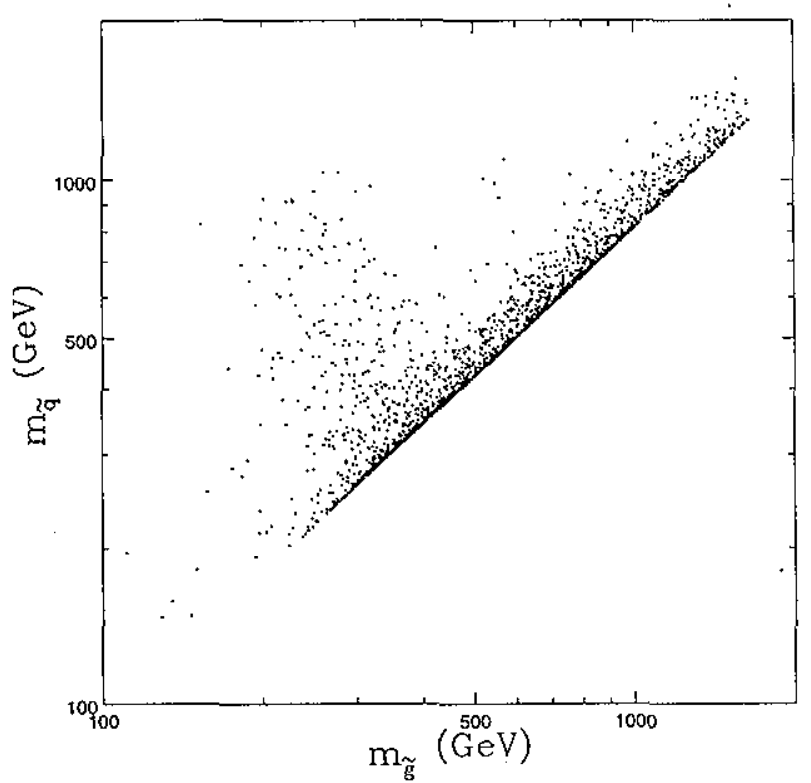

FIG. 8. The correlation between the squark $(\tilde{q})$ and gluino ( $\tilde{g})$ mass is shown for all CMSSM solutions. We have plotted $\tilde{q}=\tilde{u}_{L}$ to be representative of all the squarks. Note that relatively few solutions have $m_{\tilde{q}}=m_{\tilde{g}}$ or $m_{\tilde{q}} \gg m_{\tilde{g}}$.

The resultant weighted cross section for $\not_{T}^{\text {cut }}=$ $(0,50,75,100,125 \mathrm{GeV})$ is $(0.13 \mu \mathrm{b}, 1.3 \mathrm{pb}, 2 \mathrm{fb}, \simeq 0, \simeq$ $0)$. However, a Gaussian approximation to the resolution is not realistic, since non-Gaussian tails are known to be important. To test the sensitivity of our estimate, we have added a term to our probability distribution so that the total probability of mismeasurement from $2.58-6.00 \sigma$ is still $\simeq 1 \%$ but the probability is evenly distributed. For this case, our background estimate is $(0.13 \mu \mathrm{b}, 115 \mathrm{pb}, 1.8$ $\mathrm{pb}, 103 \mathrm{fb}, \simeq 0$ ). Because of the strong sensitivity to the non-Gaussian tail, we choose $\not_{T}^{\text {cut }}=75 \mathrm{GeV}$. This reduction of the background is conservative, since we have not utilized the altered kinematics resulting from the loss of jet energy. We further apply a cut on transverse sphericity $S_{T}>0.2$ to reduce this pure jet background to a negligible level. The other physics backgrounds we considered all have $\not_{T}$ from $Z(\rightarrow \nu \bar{\nu})+g, Z\left(\rightarrow \tau^{+} \tau^{-}\right), W(\rightarrow l \nu)+g$, and $t \bar{t}$. Since we veto events containing isolated muons and electrons with $p_{T}>15 \mathrm{GeV}, W(\rightarrow \tau \nu)$ is a large potential background. Backgrounds are also reduced by requiring that the sum $E_{T}^{j_{1}}+E_{T}^{j_{2}}+\not_{T}>300 \mathrm{GeV}$, where $j_{1}$ and $j_{2}$ are the two highest $E_{T}$ jets. Finally, we require that $\Delta \phi>0.5$ between each jet and the $Z_{T}$ direction to reduce the fake $\mathbb{H}_{T}$ from energy lost in cracks or shower fluctuations. Using these cuts, the cross sections are 5 $\mathrm{fb}, 11 \mathrm{fb}$, and $24 \mathrm{fb}$ for the $Z, W$, and $t \bar{t}$ backgrounds, respectively.

After a full simulation we find the gluino mass reach for integrated luminosities of $200 \mathrm{pb}^{-1}, 2 \mathrm{fb}^{-1}$, and 25 $\mathrm{fb}^{-1}$ to be $\sim 300 \mathrm{GeV}, \sim 350 \mathrm{GeV}$, and $\sim 400 \mathrm{GeV}$, respectively, at $10 \sigma$ signal significance (see Fig. 9). Here significance is defined as the number of signal events divided by the square root of the number of background

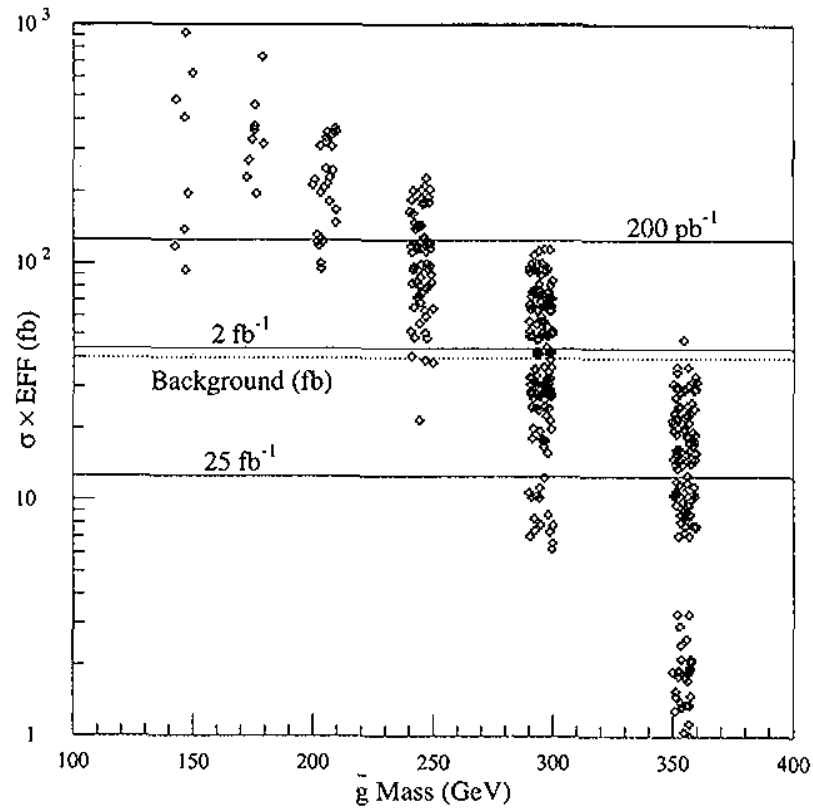

FIG. 9. The $\sigma \times E F F$ is plotted for the $\not_{2}+$ jets signal versus the gluino mass. The efficiency (EFF) is defined as the fraction of events that pass the cuts described in the text. Horizontal lines indicate the gluino mass reach of the Fermilab Tevatron at integrated luminosities of $200 \mathrm{pb}^{-1}, 2 \mathrm{fb}^{-1}$, and $25 \mathrm{fb}^{-1}$. The dotted line represents the background cross section with our cuts. The vertical banding in gluino mass is due to numerical sampling and is not physically significant.

events for events that pass the above cuts with $\not_{T}^{\text {cut }}=75$ $\mathrm{GeV}$. Of course, this is not a useful procedure unless one has reasonable knowledge of the normalization of the experimental background $\not_{T}$ distribution as well as all the real backgrounds, which we assume will occur. Note that we do not make explicit use of the signal or background shape, just the number of events above $\not_{T}^{\text {cut }}$.

We have studied several kinematic variables to see if the resulting distributions can make the signal more convincing or help us extract any information about the superpartner masses. As an example, we choose a model with $m_{\tilde{g}}=298 \mathrm{GeV}$ and $m_{\tilde{q}}=311 \mathrm{GeV}$ that has a total production cross section of $1.4 \mathrm{pb}$. The invariant mass found by summing the four-vectors of the four highest $E_{T}$ jets with the four-vector $\left(\vec{H}_{T}, \vec{\not}_{T}\right)$ is shown in Fig. 10 for the signal (dark upper bins) and the backgrounds (lower light or hashed bins). The inset figure shows the $\not_{T}$ distribution for the same processes. In the invariant mass spectrum the signal clearly peaks around $2 m_{\tilde{g}} \simeq 2 m_{\tilde{q}} \sim 600 \mathrm{GeV}$, while there is no such peak in the $\not_{x}$ distribution. Since the backgrounds from $Z$, $W$, and $t \vec{t}$ peak at lower invariant masses, one can see how the invariant mass distribution provides an effective means to separate the signal from background.

\section{E. Signals of top squark production}

The signal from top squark production has been known for some time to be quite promising $[43,23,44-46]$ since 


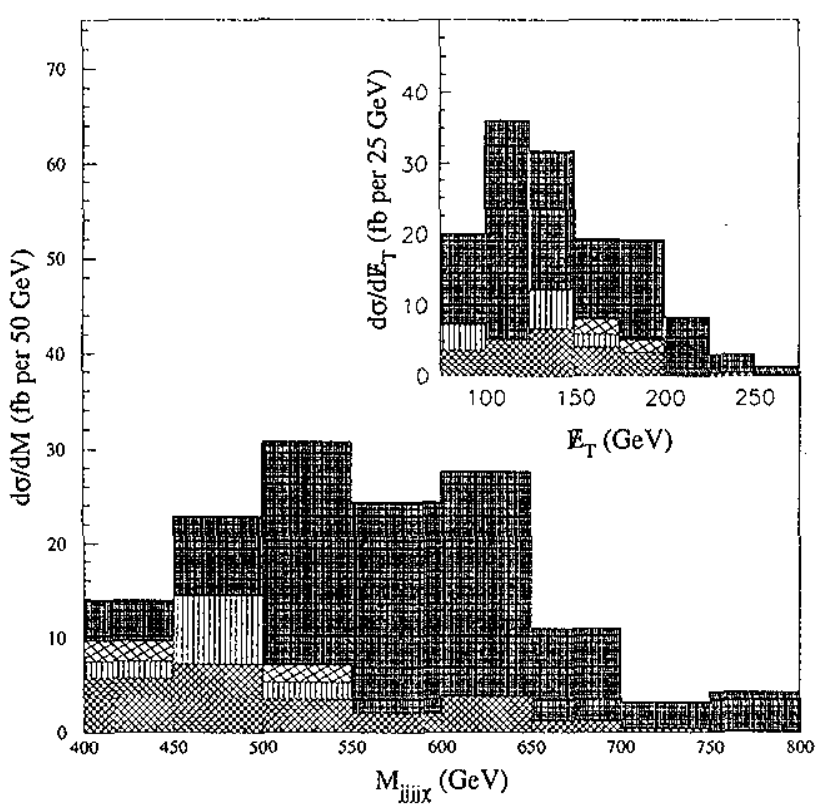

FIG. 10. The invariant mass distribution $M_{j j j j x}$ found by summing the four-vectors of the four highest $E_{T}$ jets with the four-vector $\left(\not_{T}, \vec{E}_{T}\right)$ is plotted for an example model with $m_{\tilde{g}}=298 \mathrm{GeV}$ and $m_{\tilde{q}}=311 \mathrm{GeV}$. The dark shaded region on the top is the signal, while the lower three shaded regions are the backgrounds from $Z, W$, and $t \bar{t}$ production respectively. The inset figure shows the corresponding $E_{T}$ distribution for the same model.

the lightest top squark mass eigenstate $\tilde{t}_{1}$ is generally lighter than all the other squarks. This follows from the substantial mixing between the top squark weak eigenstates $\tilde{t}_{L}$ and $\tilde{t}_{R}$ caused by a large mixing term in the top squark mass matrix proportional to the top mass. Furthermore, the large top Yukawa coupling in the running of the top squark scalar mass terms reduces the mass of the top squark with respect to the other squark masses. The result is a large mass splitting between the top squark mass eigenstates $\tilde{t}_{1}$ and $\tilde{t}_{2}$. Hence, $\tilde{t}_{1} \tilde{t}_{1}^{*}$ is more readily pair produced than the other squarks. The decay $\tilde{t}_{1} \rightarrow \tilde{\chi}_{1}^{+} b$ dominates if it is kinematically allowed, yielding a final state analogous to $t \rightarrow W^{+} b$ in the SM. For those models of the CMSSM where this decay is not allowed, we assume the decay $\tilde{t}_{1} \rightarrow \tilde{\chi}_{1}^{0} c$ with a $100 \%$ branching ratio.

A top squark search is similar to a top search if $\tilde{t}_{1} \rightarrow \tilde{\chi}_{1}^{+} b$, though we take advantage of the different kinematics to separate the top squark from the top background. The full set of backgrounds considered is $t \bar{t}, W$ $(\rightarrow l \nu)+g, Z(\rightarrow \nu \bar{\nu})+g, W^{ \pm} W^{\mp}$, and $Z\left(\rightarrow \tau^{+} \tau^{-}\right)$. Additional jet activity is generated by gluon splitting and initial and final state radiation. We classify the events into three channels: (1) dilepton, (2) W+jets, and (3) dijet. Channels (1) and (2) are distinct from a top signal because the lepton $(W+$ jets) or leptons (dilepton) have smaller $p_{T}$ and an $\not H_{T}$ inconsistent with $W$ decay. Dijet events (3) are $\not_{T}+$ jets events with no isolated leptons. We identify dilepton events by the following kinematics cuts: (1) two isolated electrons or muons with $E_{x}>10$ $\mathrm{GeV}$; (2) two or more jets, $E_{T}^{j}>20 \mathrm{GeV}$, one or two $b$ tagged; (3) $\not_{T}>25 \mathrm{GeV}$; if $25<\not_{T}<50 \mathrm{GeV}$ then an additional cut $\Delta \phi>0.3$ is applied, where $\Delta \phi$ is measured between $\vec{H}_{T}$ and any of the jets; (4) $\left|m_{\mid l^{\prime}}-m_{Z}\right|>15 \mathrm{GeV}$, and $m_{l l^{\prime}}>10 \mathrm{GeV}$ for leptons $l, l^{\prime}$; and (5) $m_{l, b}^{\max }<100$ $\mathrm{GeV}$, where $m_{l, b}^{\max }$ is the highest invariant mass between the leptons and the $b$ jets.

$W+$ jets events are defined by (1) one isolated electron or muon with $E_{T}>10 \mathrm{GeV},(2)$ two or more jets, $E_{T}^{j}>20 \mathrm{GeV}$, one or two $b$ tagged, (3) $\not_{T}>25 \mathrm{GeV}$, (4) $m_{T}<75 \mathrm{GeV}$, where the transverse mass $m_{T}$ is constructed from the lepton momentum and the $\vec{B}_{T}$, and (5) $m_{l, b}^{\max }<100 \mathrm{GeV}$, where $m_{l, b}^{\max }$ is the highest invariant mass between the leptons and the $b$ jets.

Finally, dijet events are classified by (1) two or three jets with $E_{T}^{j}>20 \mathrm{GeV}$, and no isolated leptons, (2) $\not_{T}>75 \mathrm{GeV}$, (3) $\Delta \phi_{j_{1} j_{2}}<3$, (4) $\Sigma E_{T}^{j}<150 \mathrm{GeV}$, and (5) $m_{j_{1}, j_{2}}>120 \mathrm{GeV}$, where $m_{j_{1}, j_{2}}$ is the invariant mass of the highest $\left(j_{1}\right)$ and next highest $\left(j_{2}\right) E_{T}$ jets.

For event types (1) and (2), where a $b$ tag is required, we assume an efficiency $\epsilon_{b}$ per single tag, independent of $p_{T}$ or $\eta$ of the tracks. Using $\epsilon_{b}=0.3$, the probability of tagging one or two $b$ 's is then roughly 0.5 , consistent with an upgraded Tevatron detector. For dijet events, it was shown previously that soft lepton tagging of the $c$ jet does not dramatically improve the top squark search [45], so we do not consider it here.

Our results are illustrated in Fig. 11, where the cross section folded with the detection efficiency $(\sigma \times \mathrm{EFF})$ is plotted versus the stop mass for the dilepton, $W+$ jets, and dijet channels. We find that for an integrated luminosity of $2 \mathrm{fb}^{-1}$, the top squark mass reach is up to $\sim 160$ $\mathrm{GeV}$ at $5 \sigma$ significance in the $W+$ jets channel. For 25 $\mathrm{fb}^{-1}$, the top squark mass reach is up to $\sim 200 \mathrm{GeV}$ at $5 \sigma$ significance in the $W+$ jets channel and the dilepton channel. The few models with $\tilde{t}_{1} \rightarrow \tilde{\chi}_{1}^{0} c$ are visible in the upper left corner of the dijet channel graph.

\section{F. Comment on top decays to top squark signals}

Another interesting signal of top squarks is through the decay of the top quark into a top squark and a LSP $\left(t \rightarrow \tilde{t}_{1} \tilde{\chi}_{1}^{0}\right)[43,47]$. We have analyzed all of the CMSSM solutions to find those that kinematically allow this decay and that also change the branching fraction of $t \rightarrow W^{+} b$ significantly (however, see Sec. III G). All solutions that satisfy the kinematic requirement also give $B\left(t \rightarrow \tilde{t}_{1} \tilde{\chi}_{1}^{0}\right) \lesssim 15 \%$. Since the $\tilde{\chi}_{1}^{0}$ is invisible and the top squark will be hard to see with the soft jets originating through $\tilde{t}_{1} \rightarrow \tilde{\chi}_{1}^{0} c$ decays, perhaps the only way to detect the effects of the light top squarks and LSP's is through modification of the $B\left(t \rightarrow W^{+} b\right) \simeq 1$ branching ratio.

The best way to test for the existence of these top squarks is through top squark production. Since in the $m_{t}>m_{\tilde{t}_{1}}+m_{\tilde{\chi}_{1}^{0}}$ region of parameter space the top squarks will decay primarily through a one-loop diagram to a charm and a LSP, good charm tagging would be 


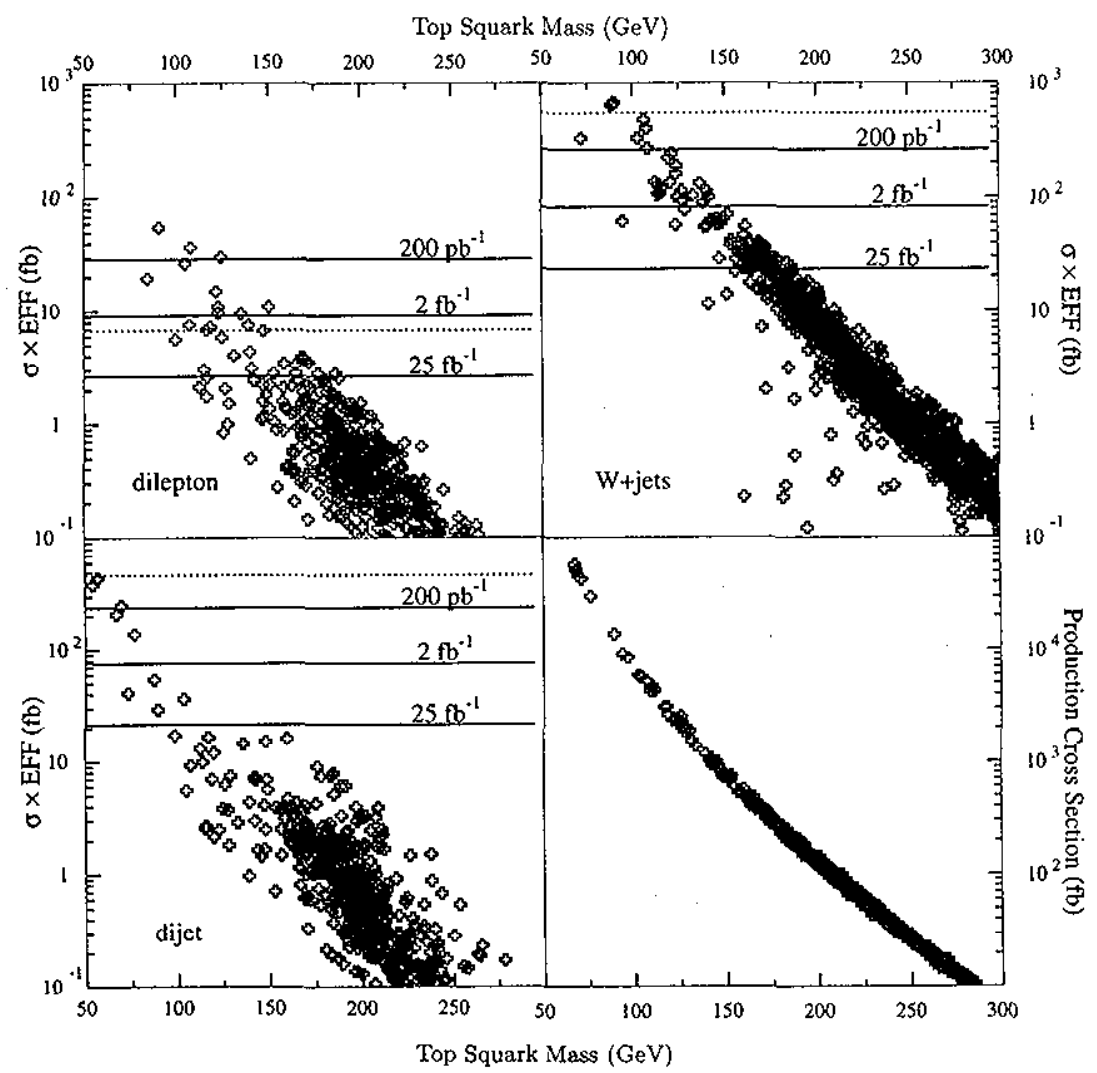

FIG. 11. The $\sigma \times$ EFF for top squark production is plotted versus the top squark mass in the dilepton, $W+$ jets and dijet channels. The efficiency (EFF) is defined as the fraction of events that pass the cuts described in the text for each channel. Horizontal lines indicate the reach of the Fermilab Tevatron at integrated luminosities of $200 \mathrm{pb}^{-1}, 2 \mathrm{fb}^{-1}$, and $25 \mathrm{fb}^{-1}$. The dotted lines represent the total background cross section for each channel with our cuts. Note the total top squark production cross section (with no cuts) is also shown for comparison. extremely helpful if this signal has a chance of being distinguished from other copious jet sources.

\section{G. Light charginos and stops beyond the CMSSM}

All analyses in this paper are based on the constrained minimal supersymmetric standard model (CMSSM) described in the Introduction. The CMSSM is more general than the SM, since it includes the SM fully, it is a consistent theory, it incorporates phenomena not explained by the SM such as the apparent unification of the gauge couplings, and it provides a derivation of the Higgs mechanism rather than assuming it as in the SM. Further, we have seen from Sec. III that the CMSSM makes a variety of testable predictions.

However, there is a hint that the CMSSM may not be entirely correct because its theoretical assumptions about supersymmetric parameters are too restrictive. The purpose of this section is to point out this possibility and the implications for supersymmetry searches at the Fermilab Tevatron.

There are two effects that are clues to go beyond the CMSSM. First, the $B(Z \rightarrow b \bar{b})$ is larger than its SM value by about $2 \sigma$. Second, the value of $\alpha_{s}$ deduced in LEP analysis from the $Z$ width is larger by about $2 \sigma$ than the value of $\alpha_{s}$ deduced other ways. While neither of these effects is of great "statistical" significance, both are based on a number of independent measurements over several years from several detectors; the errors are mostly systematic and theoretical. The possible importance of these effects is stated in Ref. [17], which finds that by including light superpartners in the analysis of the LEP data both effects can be simultaneously explained. In particular, it is remarkable that the LEP $\alpha_{s}$ measurement becomes consistent with other $\alpha_{s}$ measurements (at $\alpha_{s} \approx 0.112$ ) when light charginos and top squarks are included. Further, Ref. [17] reports a global analysis of the LEP and SLAC Linear Collider (SLC) data to ensure that no other observable is adversely affected by the light superpartner contribution (such as $\Delta \rho$ or $\left.m_{W}\right)$. One finds the supersymmetry fit is actually better than the SM fit, giving better agreement to, for example, the $R_{b}$ and $A_{L R}$ measurements. The better agreement requires both $m_{\tilde{\chi}_{1}^{ \pm}}$and $m_{\tilde{t}_{1}}$ to be less than about 100 $\mathrm{GeV}$.

Surprisingly, the properties of the chargino and top squark are sufficiently well determined by the above analysis that one is forced into concluding that the theoretical assumptions of the CMSSM cannot be fully correct. The required mixture [15] of gaugino and Higgsino in the chargino, and the left-right mixing for the top squark, are not allowed by the CMSSM, though they are fully allowed by the theory when particular assumptions about parameters in the CMSSM are relaxed. There has not been time to construct constrained models based on the nonminimal theory, but we can summarize the expected impact on the opportunity to detect superpartners at the Fermilab Tevatron.

First, and most important, the light chargino and top 
squark should be fully produced at the Fermilab Tevatron. The top squark production is predominantly via gluons and is unaffected, since the cross section is independent of $\tilde{t}_{L}, \tilde{t}_{R}$ mixing. The chargino production rate will also not be greatly affected over the parameter space. Kinematically, since charginos and stops are lighter than about $100 \mathrm{GeV}$, there is no suppression at the Fermilab Tevatron.

The signatures and detection will be different and can be more difficult. The analysis for top squark production and detection is largely the same. However, charginos that resolve the $\alpha_{\theta}$ "crisis" are more Higgsino-like than CMSSM charginos, which implies the LSP mass is closer to the chargino mass and so the resulting leptons are softer (hence, fewer leptons pass the $p_{r}$ cut). One new helpful feature does enter, in that now all top squarks are lighter than tops, so detecting top squarks in top decay is a major opportunity. The $B(t \rightarrow \tilde{t}+$ LSP $)$ has a factor of $G_{F} m_{t}^{3}$ and the usual phase space factor, plus a factor of $N_{i 4}^{2} / \sin ^{2} \beta$ that is the supersymmetric modification from the Higgsino wave functions and the top-quark-topsquark coupling. Numerically, we find $0.15 \lesssim B(t \rightarrow$ $\tilde{t}+\mathrm{LSP}) \lesssim 0.6$ for $m_{\tilde{\chi}_{1}^{ \pm}} \sim 60 \mathrm{GeV}$, to $0.05 \lesssim B(t \rightarrow$ $\tilde{t}+\mathrm{LSP}) \lesssim 0.3$ for $m_{\tilde{\chi}_{2}^{ \pm}} \sim 90 \mathrm{GeV}$.

Once the decay $t \rightarrow \tilde{t}+$ LSP has occurred, the analysis of $\tilde{t}$ decays is as before, with $\tilde{t}_{1} \rightarrow \tilde{\chi}_{1}^{+} b$ dominating if it is open, and $\tilde{t}_{1} \rightarrow \tilde{\chi}_{1}^{0} c$ otherwise. For chargino detection a new analysis is required to correctly calculate the $\mathrm{BR} \times \mathrm{EFF}$ for the trilepton signal, and the needed models have not yet been analyzed. Qualitatively, as remarked above, the leptons will be a little softer, so lowering the lepton triggers will increase the efficiency and gains by going to softer leptons may be major. The interference effect and the invisible $\tilde{\nu}_{L} \bar{\nu}$ mode may not cause such a large suppression of the leptonic branching ratio of the neutralino as in the CMSSM, possibly improving the situation for some of the parameter space.

In summary, if the LEP $R_{b}$ measurement and the $\alpha_{s}$ crisis are telling us that light charginos and top squarks exist, then they are already being produced at the Fermilab Tevatron. The challenge is to detect them. The analysis based on the CMSSM, or an extended theory, can help thinking about signature and detection efficiency, and help the interpretation if no signal is found. If light charginos and top squarks are there, high luminosity and a careful search is needed to find them.

\section{CONCLUSIONS AND COMMENTS}

We have conducted a thorough study of many possible supersymmetry signals at a luminosity upgraded Fermilab Tevatron collider. Our studies have complemented and often improved on others using our full event-level Monte Carlo based on PYTHIA-JETSET coupled with a comprehensive approach to constrained minimal supersymmetric parameter space. We conclude, consistent with earlier work, that the trilepton signal and gluino production are the most useful signals to discover supersymmetry at the Fermilab Tevatron collider. Although no limits could be placed on the mass of the lightest chargino if no signal were detected, the discovery potential at Fermilab is quite large, extending to chargino masses well beyond that which can be probed by LEP II. With integrated luminosities of $200 \mathrm{pb}^{-1}, 2 \mathrm{fb}^{-1}$ and 25 $\mathrm{fb}^{-1}$, the Fermilab Tevatron can reach to chargino masses of $140 \mathrm{GeV}, 210 \mathrm{GeV}$, and $240 \mathrm{GeV}$, respectively. Gluino searches at Fermilab will be able to detect solutions with $m_{\tilde{g}}$ up to $\sim 300 \mathrm{GeV}, \sim 350 \mathrm{GeV}$, and $\sim 400 \mathrm{GeV}$ to $10 \sigma$ significance with integrated luminosities of $200 \mathrm{pb}^{-1}, 2$ $\mathrm{fb}^{-1}$, and $25 \mathrm{fb}^{-1}$. The top squark production signal at Fermilab has a top squark mass reach up to $\sim 160 \mathrm{GeV}$ at $5 \sigma$ significance in the $W+$ jets channel with an integrated luminosity of $2 \mathrm{fb}^{-1}$. At an integrated luminosity of $25 \mathrm{fb}^{-1}$, Fermilab has a top squark mass reach up to $\sim 200 \mathrm{GeV}$ at $5 \sigma$ significance for top squark production in both the $W+$ jets and the dilepton channels.

While searches at LEP can likely find or exclude chargino and top squark masses up to nearly the beam energy $\sqrt{s} / 2$, searches at the Fermilab Tevatron have a reach $\sim 2-3$ times that of LEP II. However, the Fermilab Tevatron cannot set mass limits if no signal is found because sets of parameters exist that give smaller $\sigma \times \mathrm{BR} \times \mathrm{EFF}$ than can be detected. However, in the absence of a discovery, what matters is establishing tighter experimental constraints on the parameters; in Sec. III C we showed how a variety of information will combine to reduce the allowed parameter space so that increasingly unique and testable predictions can be made. In this pursuit, LEP II and Fermilab are comparably powerful and somewhat complementary. Searches for supersymmetry utilizing existing and upgraded collider facilities have a great potential for discovery, and if no discovery occurs at a given energy or luminosity then the results help sharpen both our understanding of the theory and predictions for a variety of other experiments.

\section{ACKNOWLEDGMENTS}

We would like to thank K. De, C. Kolda, S. Martin, and R. Watkins for helpful conversations. We are indebted to C. Kolda for the use of his renormalization group equation program. S.M. would like to thank B. Barish for support. This work was supported in part by the U.S. Department of Energy.
[1] T. Sjöstrand, Comput. Phys. Commun. 82, 74 (1994).

[2] H.-U. Bengtsson and T. Sjöstrand, Comput. Phys. Commun. 46, 43 (1987).

[3] T. Sjöstrand and M. Bengtsson, Comput. Phys. Com- mun. 43, 367 (1987).

[4] H. Baer, F. E. Paige, S. D. Protopopescu, and X. Tata, in Proceedings of the Workshop on Physics at Current Accelerators and the Supercolliders, Argonne, Illinois, 1993, 
edited by J. Hewett et al. (ANL, Argonne, 1993), p. 703, Report No. hep-ph/9305342.

[5] We note that a Monte Carlo "SUSYSM" was developed for hadron supercolliders using an interface to the PYTHIA-JETSET system in Ref. [42].

[6] F. E. Paige and S. D. Protopopescu, Report No. BNL38774,1986

[7] S. Mrenna, "Simulating Supersymmetry with PYTHIA and JETSET," Report No. CIT 68-1987 (unpublished).

[8] G. L. Kane, C. Kolda, L. Roszkowski, and J. D. Wells, Phys. Rev. D 49, 6173 (1994).

[9] H. E. Haber and G. L. Kane, Phys. Rep. 117, 75 (1985).

[10] S. P. Martin, Phys. Rev. D 46, 2769 (1992).

[11] E. Diehl, G. L. Kane, C. Kolda, and J. D. Wells, Phys. Rev. D 52, 4223 (1993).

[12] P. Sikivie, in Trends in Astroparticle Physics, Proceedings of the Workshop, Stockholm, Sweden, 1994, edited by L. Bergstrom et al. [Nucl. Phys. B (Proc. Suppl.) 43 (1995)], Report No. hep-ph/9503292 (unpublished).

[13] J.-M. Frère and G. L. Kane, Nucl. Phys. B223, 331 (1983).

[14] For a recent review, see H. Baer et al., Report No. FSUHEP-950401, hep-ph/9503479 (unpublished), and references therein.

[15] J. D. Wells, C. Kolda, and G. L. Kane, Phys. Lett. B 338, 219 (1994).

[16] M. Shifman, Mod. Phys. Lett. A 10, 605 (1995).

[17] G. L. Kane, R. G. Stuart, and J. D. Wells, Phys. Lett. B 354, 350 (1995).

[18] S. Dawson, E. Eichten, and C. Quigg, Phys. Rev. D 31, 1581 (1985).

[19] A. Bartl, H. Fraas, and W. Majerotto, Z. Phys. C 30, 441 (1986).

[20] A. Bartl, H. Fraas, and W. Majerotto, Nucl. Phys. B378, 1 (1986).

[21] J. F. Gunion and H. E. Haber, Phys. Rev. D 37, 2515 (1988).

[22] CDF Collaboration, F. Abe et al., Phys. Rev. Lett. 74, 2626 (1995).

[23] H. Baer, V. Barger, R. J. N. Phillips, and X. Tata, Phys. Lett. B 220, 303 (1989).

[24] H. Baer, C. Chen, F. Paige, and X. Tata, Phys. Rev. D 49, 3283 (1994).

[25] H. Baer, J. F. Gunion, C. Kao, and H. Pois, Phys. Rev. D 51, 2159 (1995).

[26] J. L. Lopez, D. V. Nanopoulos, X. Wang, and A. Zichichi, Phys. Rev. D 52, 142 (1995).

[27] H. Baer, C. Chen, C. Kao, and X. Tata, Phys. Rev. D 52, 1565 (1995).

[28] D. A. Dicus, S. Nandi, and X. Tata, Phys. Lett. 129B, 451 (1983); A. H. Chamseddine, P. Nath, and R. Arnowitt, ibid. 129B, 445 (1983); H. Baer, K. Hagiwara, and X. Tata, Phys. Rev. D 35, 1598 (1987); P. Nath and R. Arnowitt, Mod. Phys. Lett. A 2, 331 (1987); H. Baer and X. Tata, Phys. Rev. D 47, 2739 (1993); J. L. Lopez, D. V. Nanopoulos, X. Wang, and A. Zichichi, ibid. 48, 2062 (1993); H. Baer, C. Kao, and X. Tata, ibid. 48, 5175 (1993); H. Baer, C. Chen, F. Paige, and X. Tata, ibid. 50, 4508 (1994); H. Baer, C. Chen, R. Munroe, F. E. Paige, and X. Tata, ibid. 51, 1046 (1995).

[29] R. Barbieri, F. Caravaglios, M. Frigeni, and M. L. Mangano, Nucl. Phys. B367, 28 (1991).

[30] T. Kamon, J. L. Lopez, P. McIntyre, and J. T. White, Phys. Rev. D 50, 5676 (1994).

[31] E. Braaten and J. P. Leveille, Phys. Rev. D 22, 715 (1980).

[32] G. L. Kane, C. Kolda, and J. D. Wells, Phys. Rev. Lett. 70, 2686 (1993).

[33] R. Barbieri and G. F. Giudice, Phys. Lett. B 309, 86 (1993).

[34] J. Wu, R. Arnowitt, and P. Nath, Phys. Rev. D 51, 1371 (1995).

[35] V. Barger, M. S. Berger, P. Ohmann, and R. J. N. Phillips, Phys. Rev. D 51, 2438 (1995).

[36] CLEO Collaboration, R. Ammar et al., Phys. Rev. Lett. 71, 674 (1993).

[37] CLEO Collaboration, M. S. Alam et al., Phys. Rev. Lett. 74, 2885 (1995).

[38] A. Buras, M. Misiak, M. Münz, and S. Pokorski, Nucl. Phys. B424, 374 (1994).

[39] M. Drees and M. Nojiri, Phys. Rev. D 47, 376 (1993).

[40] L. Roszkowski, in $S U S Y$ ' 94 , Proceedings of the International Workshop on Supersymmetry and Unification of Fundamental Interactions, Ann Arbor, Michigan 1994, edited by C. Kolda and J. D. Wells (University of Michigan, Ann Arbor, 1994), p. 480.

[41] R. M. Barnett, J. F. Gunion, and H. E. Haber, Phys. Rev. D 37, 1892 (1988); H. Baer, X. Tata, and J. Woodside, ibid. 41, 906 (1990); 42, 1450 (1990); A. Bartl, W. Majerotto, B. Mösslacher, and N. Oshimo, Z. Phys. C 52, 477 (1991); H. Baer, C. Chen, F. Paige, and X. Tata, Phys. Rev. D 52, 2746 (1995).

[42] H. Baer, X. Tata, and J. Woodside, Phys. Rev. D 45, 142 (1992).

[43] J. Ellis and S. Rudaz, Phys. Lett. 128B, 248 (1983).

[44] H. Baer, M. Drees, R. Godbole, J. F. Gunion, and X. Tata, Phys. Rev. D 44, 725 (1991).

[45] H. Baer, J. Sender, and X. Tata, Phys. Rev. D 50, 4517 (1994).

[46] J. L. Lopez, D. V. Nanopoulos, and A. Zichichi, Report No. CERN-TH.7296/96, hep-ph/9406254.

[47] K. Hidaka, Y. Kizukuri, and T. Kon, Phys. Lett. B 278, 155 (1992). 